\title{
Mehrheitsfähige Klimapolitik
}

\section{Ein Kommentar zu «Whistle- blowing für das Weltklima» von Marc Thommen und Jascha Mattmann, erschienen im Januar 2021 in sui generis}

Am 13. Januar 2020 hat das Bezirksgericht Lausanne ein aufsehenerregendes Urteil gefällt, indem es zwölf Klimaaktivisten, die in einer Credit-Suisse-Filiale Tennis gespielt hatten, in allen Anklagepunkten freigesprochen hat. Das Gericht wurde scharf dafür kritisiert, dass es einen rechtfertigenden Notstand (Art. 17 StGB) angenommen hat. Einige Autoren beliessen es jedoch nicht bei der blossen Kritik, sondern brachten gleich eine alternative Begründung vor, um von der Bestrafung der Aktivisten abzusehen. Der Autor geht hier auf den Rechtfertigungs- bzw. Schuldausschlussgrund der Wahrung berechtigter Interessen ein, welcher von Marc Thommen und Jascha Mattmann ins Feld geführt wurde.

I. Zugrunde liegendes Urteil

II. Gegenstand des Kommentars

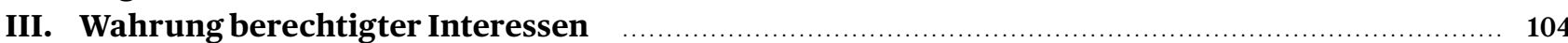

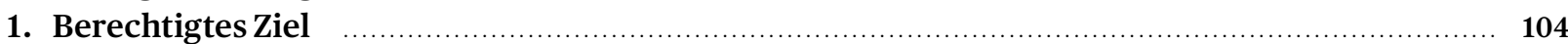

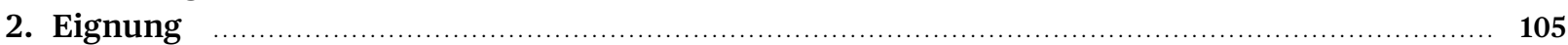

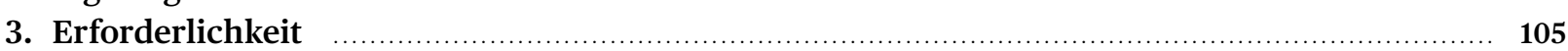

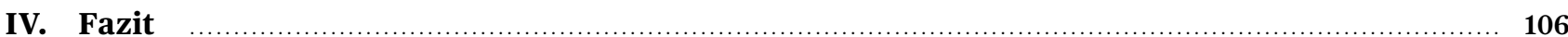

Zitiervorschlag:

ATILA STOCKER, Mehrheitsfähige Klimapolitik, sui generis 2021, S. 103 


\section{Zugrunde liegendes Urteil}

1 Durch das Urteil des Bezirksgerichts Lausanne PE19.000742/PCL/llb vom 13. Januar 2020 wurde ein Dutzend Klimaaktivisten, die in einer Credit-SuisseFiliale Tennis gespielt hatten, vom Vorwurf des Hausfriedensbruchs, der Hinderung von Amtshandlungen und der unbewilligten Demonstration freigesprochen. ${ }^{1}$ Die Aktivisten wollten mit ihrer Aktion auf die Beteiligung der Banken an der Klimaerwärmung aufmerksam machen. Der Richter nahm an, dass sämtliche Taten durch Notstand gerechtfertigt waren. Er begründete diesen Schluss damit, dass der Klimawandel eine imminente Gefahr für die Menschheit und somit auch für die Beschuldigten darstelle und der Tennis-Match das mildest mögliche geeignete Mittel war, um diese Gefahr abzuwenden. Der Entscheid wurde vielfach kritisiert. ${ }^{2}$ Das Urteil wurde durch die Folgeinstanz aufgehoben. ${ }^{3}$

\section{Gegenstand des Kommentars}

2 Am 20.Januar 2021 haben Marc Thommen und Jascha Mattmann eine Besprechung des Lausanner Bezirksgerichtsurteils unter dem Titel «Whistleblowing für das Weltklima» publiziert. Die Autoren gehen darin zuerst auf den Vorwurf des Ungehorsams gegenüber der Polizei ein und besprechen anschliessend die möglichen Rechtfertigungs- und Schuldausschlussgründe für die Tat. Sie stellen richtigerweise fest, dass entgegen dem Bezirksgericht nicht von einem rechtfertigenden Notstand ausgegangen werden kann, da die begangene Tat nicht geeignet war, das Leben der Aktivisten oder Dritter zu retten. ${ }^{4}$ Auch die Voraussetzungen eines übergesetzlichen Notstands sehen sie als nicht erfüllt an, weil die Aktion auch nicht geeignet war, kollektive Rechtsgüter wie das

1 Urteil des Bezirksgerichts Lausanne PE19.000742/PCL/llb vom 13. Januar 2020; grosses Medienecho in den darauffolgenden Tagen vgl. NZZ vom 14.Januar 2020, S. 1 (Freispruch für Klimaaktivisten) und Tages-Anzeiger vom 15.Januar 2020, S. 4 («Das Lausanner Urteil lässt aufhorchen»).

2 Vgl. LUCIEN SCHERRER, Richter im Aktivistenmodus, NZZ vom 15. Januar 2020, S. 11; HANSUELI SCHÖCHLI, «Das würde zum Chaos führen", NZZ vom 15. Januar 2020, S.13; KATHARINA FONTANA, Freipass für den Rechtsbruch, Weltwoche vom 16. Januar 2020, S.31; MARKUS MELZL, Rechtsprechung mit Zeitgeist verwechselt, Basler Zeitung vom 20. Januar 2020, S.2; GRACE SCHILD TRAPPE / FELIX SCHÖBI, Not kennt kein Gebot beim Hausfriedensbruch?, Jusletter vom 11. Mai 2020. Mit abweichender Argumentation kamen einige Autoren jedoch ebenfalls zur Straflosigkeit: ANDRÉS PAYER, Klimawandel als strafrechtlicher Notstand, sui generis 2020, S. 226; MARC THOMMEN / JASCHA MATTMANN, Whistleblowing für das Weltklima, sui generis 2021, S.13; MARTINO MONA, in: Watson vom 15. Januar 2020 (Weshalb sich Experten nach dem Freispruch der Klimaaktivisten an den Kopf fassen).

3 Urteil des Kantonsgerichts Waadt Nr.371 PE19.000742/PCL vom 22. September 2020

4 THOMMEN/MATTMANN (Fn. 2), N27; so auch TRAPPE/SCHÖBI(Fn. 2) N17; PAYER (Fn. 2), N18; Urteil des Kantonsgerichts Waadt Nr. 371 PE19.000742/PCL vom 22. September 2020 E.6.3.
Weltklima zu retten. ${ }^{5}$ In der Folge prüfen die Autoren die Wahrung berechtigter Interessen sowohl als Rechtfertigungs-als auch als Schuldausschlussgrund und kommen zum Schluss, dass die Aktivisten kein Schuldvorwurf trifft. ${ }^{6}$ Diese Argumentation soll im Folgenden näher beleuchtet und deren Schwachstellen aufgezeigt werden.

\section{Wahrung berechtigter Interessen}

Das Bundesgericht hat die Wahrung berechtigter Inter- 3 essen als Rechtfertigungsgrund anerkannt, wenn die Tat das mildest mögliche Mittel darstellt, ein berechtigtes Ziel zu erreichen (Eignung, Erforderlichkeit) und sie offenkundig weniger schwer wiegt als die gewahrten Interessen (Verhältnismässigkeit). ${ }^{7}$

\section{Berechtigtes Ziel}

Die Autoren führen aus, dass ein berechtigtes Interesse 4 vorliegt, wenn das angestrebte Ziel entweder sozial erwünscht ist oder in der Ausübung verfassungsmässiger Freiheitsrechte liegt. ${ }^{8}$ Es wird argumentiert, dass sich aus der Ratifizierung des Pariser Klimaübereinkommens ${ }^{9}$ ergibt, dass es sich beim Klimaschutz um ein sozial erwünschtes Ziel handelt. Dem ist grundsätzlich nicht zu widersprechen, jedoch ist die Formulierung des Ziels derart allgemein gefasst, dass wohl kaum singuläre, und nicht unmittelbar themenbezogene Handlungen direkt darauf hinstreben können. Vielmehr stellt sich die Frage, auf welchem Weg dieses grossmehrheitlich anerkannte Ziel umgesetzt wird. Die Klimaaktivisten im vorliegenden Fall möchten jegliche Investitionen in fossile Energien durch Schweizer Banken unterbinden. ${ }^{10}$ Ein solch radikales Vorgehen wird von der Mehrheit der Bevölkerung nicht unterstützt und es ist somit fraglich, ob es noch als sozial erwünscht betrachtet werden kann.11 Sozial erwünscht bedeutet von der Gesellschaft gewollt.

5 THOMMEN/MATTMANN (Fn. 2), N28.

6 THOMMEN/MATTMANN (Fn. 2), N36 infine.

7 BGE134IV 216 E. 6.1.

8 THOMMEN/MATtMANN (Fn. 2), N29; so auch PAYER (Fn. 2), N23.

9 Übereinkommen von Paris vom 12. Dezember 2015(SR 0.814.012), in Kraft getreten für die Schweiz am 5. November 2017.

10 «Ensemble pour [...] pousser CS à cesser ses investissements dans les énergies fossiles !» Lausanne Action Climat auf Facebook am 23. November 2018.

11 Auf eine staatliche Regulierung des Finanzplatzes Schweiz in Bezug auf klimaschädliche Investitionen wurde im neulich verabschiedeten $\mathrm{CO}_{2}$-Gesetz (Bundesgesetz über die Verminderung von Treibhausgasemissionen vom 25. September 2020, BBl 2020 7847) bewusst zugunsten der Selbstregulierung verzichtet (Ablehnung Antrag Levrat, AB S 2019925 ff.). Auch der Bundesrat zeigt in seinem Bericht «Nachhaltigkeit im Finanzsektor Schweiz: Eine Auslegeordnung und Positionierung mit Fokus auf Umweltaspekte» vom 24.Juni 2020 (insb. Ziff. 4) verschiedene Massnahmen auf, die auf die Eigenverantwortung der Finanzinstitute und der Anleger setzen und verzichtet klar auf staatliche Investitionsverbote. 
Der Wille einer Gesellschaft bestimmt sich aus dem Willen der Einzelnen, welcher sich in den politischen Mehrheiten widerspiegelt.

5 Den Autoren zufolge ist das Vorgehen der Aktivsten zudem grundrechtlich geschützt durch Art.16 und 22 BV12, sowie Art. 10 und 11 EMRK ${ }^{13}{ }^{14}$ Sie verkennen dabei die bundesgerichtliche Rechtsprechung, aus der klar hervorgeht, dass die Versammlungsfreiheit nicht das Recht beinhaltet, sich auf fremdem Boden zu versammeln. ${ }^{15}$ Dies ergibt sich bereits daraus, dass die Credit Suisse mangels Drittwirkung nicht an die genannten Grundrechte gebunden ist. ${ }^{16}$ Auch Art. 10 und 11 EMRK sehen ein solches Recht nicht vor. ${ }^{17}$

6 Das von den Aktivisten verfolgte Ziel scheint somit weder sozial erwünscht, noch liegt es in der Ausübung verfassungsmässiger Freiheitsrechte.

\section{Eignung}

7 Die Rechtfertigung der Aktivisten scheitert zusätzlich an der Eignung ihrer Handlungen, denn nur Handlungen, die geeignet sind, das berechtigte Interesse zu wahren, können gerechtfertigt sein. Es steht fest, dass der unbewilligte Aufenthalt in der Credit-Suisse-Filiale weder geeignet ist, die Klimaerwärmung zu stoppen, noch geeignet ist, einen Investitionsstopp für fossile Energien herbeizuführen. Die Autoren umgehen diese Frage, indem sie prüfen, ob der Tennis-Match geeignet war, die Aufmerksamkeit der Öffentlichkeit auf die Klimapolitik zu richten. ${ }^{18}$ Sie legen jedoch nicht dar, inwieweit es sich beim Aufmerksammachen der Öffentlichkeit um ein sozial erwünschtes oder grundrechtlich geschütztes Ziel handelt. Dies geht nicht aus dem Pariser Klimaabkommen hervor, das darauf abzielt, dass die Mitgliedstaaten einen direkten Beitrag zum Klimaschutz leisten und nicht bloss öffentlich darauf aufmerksam machen.

\section{Erforderlichkeit}

8 Bei der Frage nach der Erforderlichkeit der Handlungen ist zu prüfen, ob mildere, insbesondere legale Mittel hät-

12 Bundesverfassung der Schweizerischen Eidgenossenschaft vom 18. April 1999 (BV; SR 101).

13 Konvention zum Schutze der Menschenrechte und Grundfreiheiten, abgeschlossen in Rom am 4. November 1950 (EMRK; SR 0.101).

14 THOMMEN/MATTMANN (Fn. 2), N30.

15 «La liberté de réunion [...] ne comprend en tout cas pas le droit de s'assembler sur le fonds d'autrui.» (BGE 97 I 911 E. 3a).

16 ULRICH HÄFELIN / WALTER HALLER / HELEN KELLER / DANIELA THURNHERR, Schweizerisches Bundesstaatsrecht, 10. Auflage, Zürich 2020, N279.

17 Vgl. Guide on Article11 of the European Convention on Human Rights, herausgegeben vom Europäischen Gerichtshof für Menschenrechte am 31. Dezember 2020, N21.

18 THOMMEN/MATTMANN (Fn. 2), N31. ten gewählt werden können, um das berechtigte Ziel zu erreichen. ${ }^{19}$ «In einem demokratischen Rechtsstaat sind politische und ideelle Anliegen grundsätzlich auf politischem Wege bzw. auf dem Rechtsweg zu verfolgen» ${ }^{20}$, hält das Bundesgericht zutreffend fest. Die Autoren folgen hier der Argumentation des Bezirksrichters, dass die Aktivisten bereits alle legalen Mittel, insbesondere den Weg über die Politik, ausgeschöpft hätten und diese erfolglos geblieben seien. ${ }^{21}$ Es ist jedoch fraglich, ob dies in Bezug auf einen Investitionsstopp in fossile Energien tatsächlich zutrifft. Das Bezirksgericht führt aus, dass der Bundesrat ungenügend auf entsprechende Vorstösse von Bundesparlamentariern eingegangen ist. ${ }^{22}$ Es wurden etliche Interpellationen zu diesem Thema eingereicht, welche allesamt vom Bundesrat ordnungsgemäss beantwortet wurden. ${ }^{23}$ Das einzige eingereichte Postulat wurde vom Nationalrat abgelehnt und zwei Motionen wurden abgeschrieben, weil sie nicht innert zwei Jahren behandelt wurden, und von den entsprechenden Parlamentsmitgliedern nicht wieder aufgegriffen wurden, wodurch sich der Bundesrat nie damit beschäftigen musste. ${ }^{24}$ Zusätzlich hätte das Anliegen auf dem Weg der parlamentarischen Initiative oder der Volksinitiative durchgesetzt werden können. Es ist falsch, der Regierung hier einen Vorwurf zu machen, vielmehr muss man zum Schluss gelangen, dass die politischen Mittel nicht genügend ausgeschöpft wurden.

Selbst wenn man davon ausgeht, dass alle politischen 9 Möglichkeiten erfolglos ausgeschöpft wurden, "gibt [dies] Letzteren [den Aktivisten] kein Recht, ihre Anliegen mit strafbaren Methoden zu verfolgen.» ${ }^{25}$ Wenn radikale Klimaschutzmassnahmen, wie sie die Aktivisten fordern, auf dem Weg der politischen Mitsprache nicht

\footnotetext{
19 BGE134IV 216 E. 6.1.

20 BGE129IV6E.3.1.

21 THOMMEN/MATTMANN (Fn. 2), N32.

22 Urteil des Bezirksgerichts Lausanne PE19.000742/PCL/llb vom 13. Januar 2020, S. 54.

23 Interpellation Thorens (15.3613) « $\mathrm{CO}_{2}$-Fussabdruck von Investitionen senken (Desinvestition aus fossilen Energieträgern oder «Divestment)). Handeln im Hinblick auf die Klimakonferenz in Paris» vom 17.Juni 2015; Interpellation Girod (15.4109) «Klimaschutz und Finanzplatzrisiken. Wie setzt die Schweiz die Empfehlungen um?» vom 8. Dezember 2015; Interpellation Chevalley (17.3120) «Was tut die Schweiz für nachhaltige Finanzen?» vom 15. März 2017; Interpellation Jans (17.3914) «Wann wird der Finanzsektor in die Pflicht genommen?» vom 29. September 2017; Interpellation Jans (17.3915) «Wann kontrolliert die Finma die Klimarisiken?» vom 29. September 2017; Interpellation Thorens (17.3946) «Ein Nachhaltigkeitstest für die künftigen Regulierungen der Finanzmärkte?» vom 29. September 2017; Interpellation Landolt (17.4104) «Zukunftsorientiertes Investieren" vom 13. Dezember 2017.

24 Postulat Chevalley (18.3589) «Instrumente zur Überprüfung nachhaltiger Finanzen» vom 14.Juni 2018; Motion Masshardt (18.3918) «Klimastrategie für den Schweizer Finanzmarkt» vom 27. September 2018; Motion Jans (18.3974) «Den grössten Klimaheizern den Geldhahn zudrehen» vom 27. September 2018.

25 BGE129IV6 E.3.1 
durchgesetzt werden können, so zeigt dies, dass sie nicht von der Mehrheit der stimm- und wahlberechtigten Bevölkerung unterstützt werden. Dies wiederum lässt darauf schliessen, dass diese Massnahmen nicht sozial erwünscht sind. Die Argumentation der Autoren ist widersprüchlich, wenn sie die Forderungen der Aktivisten einerseits als sozial erwünscht, aber andererseits als demokratisch nicht durchsetzbar, also nicht mehrheitsfähig betrachten.

\section{Fazit}

Die Wahrung berechtigter Interessen ist im vorliegenden Fall weder als Rechtfertigungs-, noch als Schuldausschlussgrund anwendbar, da die Aktivisten (1) kein berechtigtes Interesse verfolgen, (2) ihre Aktion nicht geeignet war, das verfolgte Interesse zu erreichen, und (3) der Grundsatz der Erforderlichkeit nicht erfüllt wurde, da mildere Mittel hätten gewählt werden können.

Die Argumentation nach Thommen und Mattmann wür- 11 de darauf hinauslaufen, dass zu illegalen Mitteln gegriffen werden darf, wenn ein Ziel auf politischem Wege nicht erreicht werden kann. Dahinter versteckt sich der Gedanke, dass es erstrebenswerte Ziele gibt, die von der Mehrheit der Bevölkerung nicht erkannt würden. Dies steht dem Konzept der Demokratie entgegen, da es sich in einer Demokratie gerade aus der Mehrheit ergibt, was die erstrebenswerten Ziele und der richtige Weg sind, um diese zu erreichen. Es bleibt unumstritten, dass auch dem Volkswillen Grenzen gesetzt sind, namentlich durch das ius cogens. Eine lasche Klimapolitik steht damit jedoch nicht im Widerspruch. 